\title{
Robot Excitation Trajectories for Dynamic Parameter Estimation using Optimized B-Splines
}

\author{
Wolfgang Rackl, Roberto Lampariello and Gerd Hirzinger
}

\begin{abstract}
In this paper we adressed the problem of finding exciting trajectories for the identification of manipulator link inertia parameters. This can be formulated as a constraint nonlinear optimization problem. The new approach in the presented method is the parameterization of the trajectories with optimized B-splines. Experiments are carried out on a 7 joint Light-Weight robot with torque sensoring in each joint. Thus, unmodeled joint friction and noisy motor current measurements must not be taken into account. The estimated dynamic model is verified on a different validation trajectory. The results show a clear improvement of the estimated dynamic model compared to a CAD-valued model.
\end{abstract}

\section{INTRODUCTION}

The knowledge of the dynamic model of a manipulator is a key issue in many robotic applications. The controller performance and its design in general depends on the robot model and can be improved with it. Most manufacturers focus on the quality of the kinematics in order to secure sufficient positioning accuracy. The dynamic properties are normally generated from CAD data which are not identical to the real robot. Furthermore, in the field of motion planning an accurate model is essential to obtain useful solutions. Especially the mass and center of mass of each link are important to realize exact gravitation compensation control. The identification of the inertial parameters like mass, center of mass and moments of inertia requires experiments with special exciting trajectories. Most industrial robots only use joint position sensors. The applied moments in each joint, required for the parameter estimation, must be calculated via the motor current and the position signal and the joint friction must be included in the model.

In this paper we present an alternative approach for finding exciting trajectories for the identification of robot link inertia parameters. To solve a constraint nonlinear optimization problem we use B-splines for the parameterization of the trajectories. The experiments are carried out on the 7 joint DLR Light-Weight Robot which is equipped with torque sensors in each joint. The estimated model is verified on a different validation trajectory and the accuracy is compared with a CAD-valued model.

\section{A. Related Work}

The problem of finding good excitation trajectories is a common problem in the field of parameter identification and different approaches have been presented in the literature. In [1] the trajectory is optimized online by minimizing the condition number of the observation matrix. In [2], [3], [4] and [5] the condition number of the dynamic energy model is optimized off-line to obtain a set of optimum points. In a second step, these optimum points are interpolated using fifth-order polynomial functions assuming zero initial and final velocity and acceleration. The interpolation is obtained by a further optimization problem to fit acceleration limits in all joints. Following, a third optimization to fit all velocity constraints. Finally, the position constraints are verified to fit all position limits. If they do not, a new sequence of optimum points with smaller constraints must be started. This trialand-error algorithm has some uncertainty in terms of finding practicable solutions.

A different approach for the design of exciting trajectories was presented in [6], based on a statistical framework, which is also performed off-line. It differs both in the parameterization of the trajectory and in the optimization criterion. The trajectory for each joint consists of a finite sum of harmonic sine and cosine functions, which comes with important advantages: it allows to improve the signal-to-noise ratio of the measured data by time-domain data averaging, as well as allowing to specify the bandwidth of the excitation trajectories in terms of avoiding modeling errors arising from robot flexibility. Further it allows the calculation of the joint velocities and accelerations analytically. The optimization criterion here is the minimization of the uncertainty on the estimated parameters via the covariance matrix.

\section{FORMULATION OF THE IDENTIFICATION PROBLEM}

\section{A. The DLR Light-Weight Robot}

The DLR Light-Wight Robot (LWR) is a serial chain robot with 7 rotational joints. With its ratio of payload-to-total weight of one, it is able to handle payloads of $14 \mathrm{~kg}$ over its complete dynamic range. Since the robot is equipped in each joint with a position sensor for the motor and for the joint, as well as a sensor for the joint torques, it can be controlled in position, velocity and torque mode. Especially for the torque controlled mode, it is important to use a precise dynamic model to improve the performance of the control algorithm. Due to the fact that the torque sensors are placed on the output side of the joint, no friction must be identified for the purpose of the identification. The geometric model of the robot given by CAD data is assumed to be sufficiently exact. The kinematics of the robot is shown in Fig. 1.

\section{B. Formulation of the Dynamic Model}

For the parameter identification different models were used in the literature [1], [5], [7], [8], [9], [10], [11], [12], [13] and [14]. As discussed in [3] we use here the inverse dynamic model. An effective algorithm for estimating inertial 


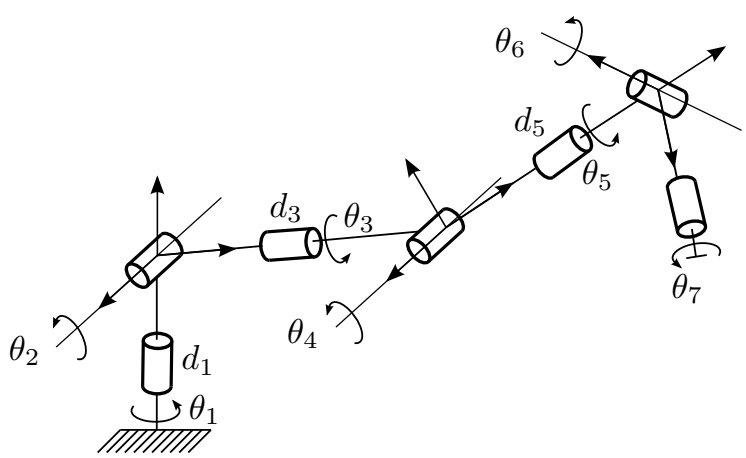

Fig. 1. Kinematic configuration of the DLR LWR 4

parameters of manipulator links and loads is presented in [7] and [10] and is recalled in this section shortly.

By using the Newton-Euler approach all acting forces and torques are calculated for each manipulator link separately. The equation for each link can be written in parameter linear form as

$$
\mathbf{w}_{i i}=\mathbf{A}_{i} \Phi_{i}
$$

with $\mathbf{w}_{i i}$ as vector of forces and torques acting on joint $i$ caused only by the movement of joint $i$. The kinematic matrix $\mathbf{A}_{i}$ describes the motion of link $i$ and is a function of the geometry, joint angle $\theta_{i}$, joint velocity $\dot{\theta}_{i}$ and the joint acceleration $\ddot{\theta}_{i}$. The parameter vector $\boldsymbol{\Phi}_{i}$ consists of the inertial parameters

$$
\begin{aligned}
\boldsymbol{\Phi}_{i}=\left[M_{i}, M X_{i}, M Y_{i}, M Z_{i},\right. & X X_{i}, X Y_{i}, \cdots \\
& \left.\cdots X Z_{i}, Y Y_{i}, Y Z_{i}, Z Z_{i}\right]^{T}
\end{aligned}
$$

where

- $M_{i}$ is the mass of link $i$

- $M X_{i}, M Y_{i}, M Z_{i}$ are the components of the first moments and

- $X X_{i}, X Y_{i}, X Z_{i}, Y Y_{i}, Y Z_{i}, Z Z_{i}$ are the components of the inertia matrix of joint $i$.

The total wrench of forces and torques on joint $i$ is the sum of all wrenches $\mathbf{w}_{i, j}$ distal to joint $i$. Since only the torques in the joint axis can be measured, each joint wrench must be reduced onto its rotation axis. The matrix expression of the serial chain manipulator comes to

$$
\left[\begin{array}{c}
{ }^{1} \tau_{1} \\
{ }^{2} \tau_{2} \\
\vdots \\
{ }^{n} \tau_{n}
\end{array}\right]=\left[\begin{array}{cccc}
\mathbf{K}_{11} & \mathbf{K}_{12} & \cdots & \mathbf{K}_{1 n} \\
\mathbf{0} & \mathbf{K}_{22} & \cdots & \mathbf{K}_{2 n} \\
\vdots & \vdots & \ddots & \vdots \\
\mathbf{0} & \mathbf{0} & \cdots & \mathbf{K}_{n n}
\end{array}\right]\left[\begin{array}{c}
{ }^{1} \boldsymbol{\Phi}_{\mathbf{1}} \\
{ }^{2} \boldsymbol{\Phi}_{2} \\
\vdots \\
{ }^{n} \boldsymbol{\Phi}_{n}
\end{array}\right]
$$

or

$$
\tau=\mathbf{K} \Psi
$$

where $\tau$ is the $n \times 1$ vector of joint torques with $n$ links, $\mathbf{K}$ is the so called observation matrix or regressor matrix and $\boldsymbol{\Psi}$ is the vector of all joint parameters $\boldsymbol{\Phi}_{i}$.

\section{Estimation Procedure}

Since the system of equations in (2) has $10 n$ unknown parameters but only $n$ equations, $N$ measurements along a trajectory are required to set up an over-determined system of linear equations

$$
\mathbf{Y}=\Pi \Psi+\rho
$$

with the observation matrix or regressor matrix $\Pi$ and the vector of all measured torques $\mathbf{Y}$ given by

$$
\boldsymbol{\Pi}=\left[\begin{array}{c}
\mathbf{K}(1) \\
\mathbf{K}(2) \\
\vdots \\
\mathbf{K}(N)
\end{array}\right] ; \mathbf{Y}=\left[\begin{array}{c}
\boldsymbol{\tau}(1) \\
\boldsymbol{\tau}(2) \\
\vdots \\
\boldsymbol{\tau}(N)
\end{array}\right]
$$

and the vector of errors $\rho$ between the measured torques and the model predicted torques.

As suggested in [3] a weighted least-squares optimization can be used to estimate the parameters in (4)

$$
\boldsymbol{\Psi}=\underset{\Phi}{\operatorname{Arg} \cdot \min }\|\rho\|^{2}=\left(\boldsymbol{\Pi}^{T} \boldsymbol{\Pi}\right)^{-1} \boldsymbol{\Pi}^{T} \mathbf{Y}=\boldsymbol{\Pi}^{+} \mathbf{Y}
$$

Since the focus of this paper is the generation of exciting trajectories for the dynamic parameter identification, the procedure was split into two parts: firstly, the identification of the base parameters out of $M_{i}, M X_{i}, M Y_{i}$ and $M Z_{i}$ - here called static parameters - and secondly, the identification of the components of the inertia matrix of each link, here called dynamic parameters with the static parameters assumed known. The advantage on this sequential identification is that for the static identification only measurements with fixed poses are necessary, and as such uncertainties in generating the joint velocities and accelerations are avoided. Furthermore, the optimization problem to generate exciting poses depends only on joint positions and the number of parameters to be optimized for the generation of the exciting trajectories is significantly less than for the complete identification. The identification of the static parameters was done prior to the identification of the dynamic parameter and will not be described here.

The set of minimal dynamic parameters $\boldsymbol{\Phi}_{d y n}$ - the base parameters - can be determined using the simple closed-form rules of [12],[15] and [16]. With the known static parameters, the equations of motion can be extended to

$$
\mathbf{Y}-\boldsymbol{\Pi}_{\text {stat }} \boldsymbol{\Psi}_{\text {stat }}=\boldsymbol{\Pi}_{d y n} \boldsymbol{\Psi}_{d y n}+\rho
$$

and the estimation in (6) comes to

$$
\begin{aligned}
\boldsymbol{\Psi}_{d y n} & =\left(\boldsymbol{\Pi}_{\text {dyn }}^{T} \boldsymbol{\Pi}_{\text {dyn }}\right)^{-1} \boldsymbol{\Pi}_{\text {dyn }}^{T}\left(\mathbf{Y}-\boldsymbol{\Pi}_{\text {stat }} \boldsymbol{\Psi}_{\text {stat }}\right) \\
& =\boldsymbol{\Pi}^{+}{ }_{\text {dyn }}\left(\mathbf{Y}-\boldsymbol{\Pi}_{\text {stat }} \boldsymbol{\Psi}_{\text {stat }}\right)
\end{aligned}
$$

\section{Sensitivity of the Solution and Degree of Estimation}

To evaluate the estimated parameters, several criteria were proposed in the literature [2],[3],[6] and [12]. For the level of excitation of each parameters, we use the 2norm condition number, which depends on the smallest and the biggest singular value of the regressor matrix. The smaller the condition number, the higher the excitation of 
each parameter and thus disturbances and noise decrease their impact on the estimation. Furthermore, the accuracy of an estimated parameter can be expressed with the relative standard deviation. Hereby it is assumed that the observation matrix $\Pi$ is deterministic and that the torque error $\rho$ is a zero-mean additive independent Gaussian noise with $\sigma_{\rho}$ as standard deviation. The variance-covariance matrix $\mathbf{C}_{\rho}$ can be calculated as in [12] with

$$
\mathbf{C}_{\rho}=E\left(\rho \rho^{T}\right)=\sigma_{\rho}^{2} \mathbf{I}_{\mathbf{r}}
$$

$E$ is called the expectation operator, $\mathbf{I}_{\mathbf{r}}$ is the $(r \times r)$ identity matrix and $\sigma_{\rho}$ is given by the unbiased estimation

$$
\sigma_{\rho}^{2}=\frac{\left\|\boldsymbol{\tau}-\boldsymbol{\Pi} \boldsymbol{\Phi}_{\boldsymbol{e s t}}\right\|^{2}}{(r-c)}
$$

with

$$
\begin{array}{lll}
r & = & \text { total number of equations } \\
c & = & \text { number of parameters }
\end{array}
$$

The variance-covariance matrix of the estimation error is given as

$$
\begin{aligned}
\mathbf{C}_{\boldsymbol{\Phi}_{\text {est }}} & =\mathbf{E}\left[\left(\boldsymbol{\Phi}-\boldsymbol{\Phi}_{\text {est }}\right)\left(\boldsymbol{\Phi}-\boldsymbol{\Phi}_{\text {est }}\right)^{T}\right]= \\
& =\boldsymbol{\Pi}^{+} \mathbf{C}_{\rho}\left(\boldsymbol{\Pi}^{+}\right)^{T}=\sigma_{\rho}^{2}\left(\boldsymbol{\Pi}^{T} \boldsymbol{\Pi}\right)^{-1}
\end{aligned}
$$

With the variance-covariance matrix we can extract the standard deviation of the estimation error $\sigma_{\Phi_{e s t, j}}$ and its relative value $\sigma_{\Phi_{e s t, j r}}$ as the $(j, j)$ element of $\mathbf{C}_{\boldsymbol{\Phi}_{\text {est }}}$

$$
\sigma_{\Phi_{e s t, j}}=\sqrt{\mathbf{C}_{\Phi_{\text {est }}}(j, j)}
$$

and

$$
\sigma_{\Phi_{e s t, j r}}=100 \frac{\sigma_{\Phi_{e s t, j}}}{\left|\Phi_{e s t, j}\right|}
$$

In [3] and [12] a parameter is assumed to be poorly identified if the relative error deviation is in the area between 5 to $15 \%$ or above.

\section{GENERATION OF OPTIMAL ROBOT EXCITATION TRAJECTORIES}

The parameter excitation is addressed here as a nonlinear optimization problem.

\section{A. Optimization Problem Formulation}

The optimization problem contains a configuration space $C$ of dimensions $C(\boldsymbol{\theta}) \subseteq \Re^{n}$, where $n$ is the number of robot joints and $\boldsymbol{\theta}$ the vector of joint positions. The time interval for the spline is $t_{f}$. The optimization problem can be formulated as

$$
\min _{\theta, t_{f}} \Gamma
$$

where $\Gamma$ is a predefined cost function and is described below.
1) Cost function: The cost function for the nonlinear optimization problem is defined as the sum of two scalars and is defined in detail as

$\Gamma=\xi_{1} \lambda\left(\boldsymbol{\Pi}_{d y n} \operatorname{diag}\left(\boldsymbol{\Phi}_{C A D}\right)\right)+\xi_{2}\left(\sum_{i=0}^{n} \sum_{j=0}^{h} \tau_{i, j}\right)^{-1}$

where the first summand is the condition number $\lambda$ of the product of observation matrix $\boldsymbol{\Pi}_{d y n}$ and the diagonal matrix containing the known CAD values of the respective parameters as discussed in [4]. The second summand is the reciprocal of the sum of the joint torques for all joints over preselected points $h$ of the trajectory. It was chosen to improve the signal-to-noise ratio of the measurements. $\xi_{i}$ are scaling factors for each criterion.

2) Constraints: Due to the constraint of collision avoidance, two different bounds for the end-effector position are built. Firsty, the end-effector must not collide with its environment and secondly, a self collision must be avoided. Thus the workspace for the robot is defined for simplicity as a cubic box around the manipulator with the conditions

$$
x_{\min } \leq x_{e e} \leq x_{\max }
$$

with $x=(x, y, z)$ the coordinates in the cartesian space.The constraint for the self collision is simply defined as a sphere around the manipulator base with the radius $r_{\text {scoll }}$

$$
r_{\text {scoll }} \leq x_{e e}
$$

A further constraint must be defined for the robots joint limits. So the given intervals must not be exceeded for any time during the trajectory for each joint

$$
h_{i, \min } \leq h_{i} \leq h_{i, \max }, i=1, \ldots, n
$$

where $h$ stands for $x \subset\left\{\theta_{i}, \dot{\theta}_{i}, \ddot{\theta}_{i}, \tau_{i}\right\}$.

\section{B. Parameterization of the Trajectory}

Using polynomials for interpolating trajectory points, a change of one parameter will change the complete interval of the trajectory. Trajectories built with B-splines can be adjusted locally without affecting the rest of the trajectory. Due to the fact that the trajectories for the parameter identification must be defined in the robots joint space B-splines were used here. The use of B-splines and the way how they can be manipulated, in terms of searching for exciting trajectories will be described below. Fig. (2) shows an example of a B-spline interpolated via $n+1$ points.

A B-spline is defined between the knot interval from $u_{\text {min }}$ to $u_{\max }$ as

$$
\mathbf{s}(u)=\sum_{j=0}^{m} p_{j} B_{j}^{p}(u), \quad u_{\min } \leq u \leq u_{\max }
$$

where $p_{j}$ are the scalar control points, $B_{j}^{p}$ are the basis functions of degree $p$ and $m$ is the number of control points. The variable $u$ defines the knot vector. To avoid high forces and moments on the robot during the identification process, 


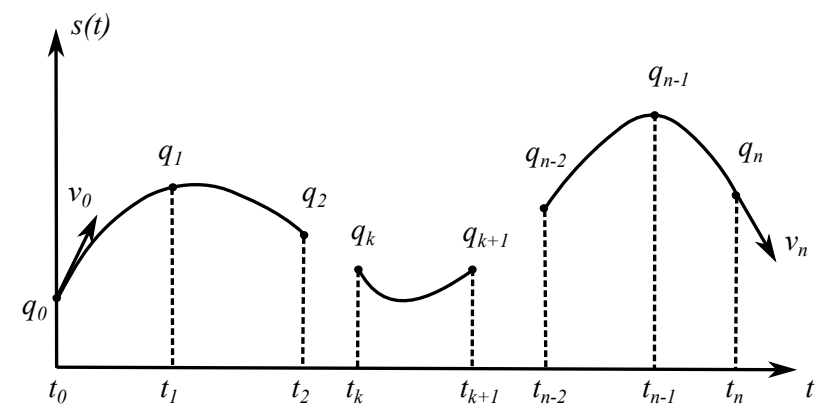

Fig. 2. Spline trajectory via $\mathrm{n}+1$ points [17]

at least a continuous jerk is defined here. Thus basis functions of degree $p=4$ are used as suggested in [17].

Since we set up the splines in the joint space of the manipulator the dimension of the spline can be reduced to the time dimension.

$$
\mathbf{s}(t)=\sum_{j=0}^{m} p_{j} B_{j}^{p}(t), \quad t_{\min } \leq t \leq t_{\max }
$$

Now, with the given points $q_{k}, k=0, \ldots, n$ which should be interpolated at the time instants $t_{k}$, the unknown control points $p_{j}, j=0, \ldots, m$ must be found under the condition

$$
s\left(t_{k}\right)=q_{k}, \quad k=0, \ldots n
$$

At first, it is important to define the knot vector $u$. In the case of spline order $p=4$ it should be definded as

$$
\begin{array}{r}
\mathbf{u}=\left[t_{0}, \ldots, t_{0}, \frac{t_{0}+t_{1}}{2}, \ldots, \frac{t_{k-1}+t_{k}}{2}, \ldots,\right. \\
\left.\ldots, \frac{t_{n-1}+t_{n}}{2}, t_{n}, \ldots, t_{n}\right]
\end{array}
$$

The number of the beginning and ending knots must be $p+1$ and the total number of knots is defined with

$$
n_{k n o t}=n+2 p+1
$$

and the number of unknown control points are

$$
m=(n+1)+p
$$

In order to obtain the unknown control points $p_{j}$, a linear system by summarizing the $n+1$ equations can be build for each point $q_{k}$ at the time $t_{k}$

$$
q_{k}=\left[B_{0}^{p}\left(t_{k}\right), B_{1}^{p}\left(t_{k}\right), \ldots, B_{m-1}^{p}\left(t_{k}\right), B_{m}^{p}\left(t_{k}\right)\right] \mathbf{p}
$$

with

$$
\mathbf{p}=\left[p_{0}, p_{1}, \ldots, p_{m-1}, p_{m}\right]^{T}, k=0, \ldots, n
$$

For the identification trajectory some boundary conditions should be fulfilled like zero initial and final velocity and acceleration:

$$
\begin{aligned}
s^{(1)}\left(t_{0}\right) & =v_{0} \\
s^{(2)}\left(t_{0}\right) & =a_{0} \\
s^{(1)}\left(t_{n}\right) & =v_{n} \\
s^{(2)}\left(t_{n}\right) & =a_{n}
\end{aligned}
$$

To add these constraints, the $i^{t h}$ derivatives of a spline can be written in general as

$$
\begin{aligned}
& s^{(i)}\left(t_{k}\right)=\left[B_{0}^{p^{(i)}}\left(t_{k}\right), B_{1}^{p^{(i)}}\left(t_{k}\right), \ldots,\right. \\
&\left.\ldots, B_{m-1}^{p^{(i)}}\left(t_{k}\right), B_{m}^{p^{(i)}}\left(t_{k}\right)\right] \mathbf{p}
\end{aligned}
$$

with the $i^{t h}$ derivative of the basis function $B_{j}^{p^{(i)}}\left(t_{k}\right)$ (for the calculation, see [17])

The complete spline can be summarized in matrix form as

$$
\mathbf{A p}=\mathbf{c}
$$

with the matrix of basis functions $\mathbf{A}$ and the given trajectory points

$$
\mathbf{c}=\left[q_{0}, v_{0} . a_{0}, q_{1}, \ldots, q_{n-1}, a_{m}, v_{n}, q_{n}\right]^{T}
$$

Finally, the control points $p$ can be obtained by solving the determined linear system of equations (31) and the complete B-spline can be built with the given constraints (for more detail, see [17]).

\section{Method of Solution}

The described optimization problem is solved as a nonlinear programming problem (NPL) by satisfying the inequality constraints described above at all selected trajectory points. For solving the NPL a Sequential Quadratic Programming algorithm from Matlab was used.

In each iteration step, at the beginning a new B-spline trajectory is calculated up to its second derivative. Out of the new B-spline a total number of $h$ points are selected and both the weighted condition number and the joint torques are calculated. The extraction of the discrete trajectory points is equally distributed along the complete B-spline. This is be done to minimize the computation effort during the optimization process. Furthermore, the time resolution of the B-spline trajectory is chosen greater for the same reason compared to the final solution of the B-spline.

To find a global optimum of the solution we run the optimization serveral times with ramdom initial guesses for the starting parameters.

\section{EXPERIMENTAL RESULTS}

The identification was made for the LWR 4 and the experimental conditions are described below. For the workspace following boxes are defined in $[\mathrm{m}]$

$$
\begin{aligned}
-1.3 \leq x_{e e} & \leq 1.5 \\
-1.3 \leq y_{e e} & \leq 1.5 \\
0.3 \leq z_{e e} & \leq 1.5 \\
r_{\text {scoll }} & \leq 0.3
\end{aligned}
$$

and for the joint position limit constraints in $\left[{ }^{\circ}\right]$

$$
\begin{array}{rc}
-170 \leq \theta_{i} \leq 170, & i=1,3,5 \\
-120 \leq \theta_{i} \leq 120, & i=2,4 \\
-45 \leq \theta_{i} & \leq 80, \quad i=6 \\
-30 \leq \theta_{i} & \leq 60, \quad i=7 \\
0.3 & \leq x_{e e}
\end{array}
$$


The vector for the joint velocity and acceleration constraints in $[\mathrm{rad} / \mathrm{s}]$ or $\left[\mathrm{rad} / \mathrm{s}^{2}\right]$ are

$$
\begin{gathered}
\dot{\theta}_{\min , \max }= \pm[1.88,1.88,2.22,2.22,3.56,3.20,3.20] \\
\ddot{\theta}_{\min , \max }= \pm[5.23,5.23,8.72,8.72,8.72,17.45,17.45]
\end{gathered}
$$

The torque limits are given in $[\mathrm{Nm}]$

$$
\tau_{\min , \max }= \pm[200,200,100,100,100,30,30]
$$

A globally optimized trajectory comes to a condition number of $\lambda=22.98$ and it is shown for axis two in Fig. (3). Random trajectories without optimization show condition numbers of about $\lambda \approx 120$. The dashed lines in the figure depict the joint limits. The markers on the position graph show the optimized position points. Further, the initial and final conditions for the velocity and accelerations are fullfiled. In order to improve the signal-to-noise ratio, the identification trajectory was optimized with a known payload. The payload data are obtained from a CAD model assumed to be highly precise due to its simple geometry.
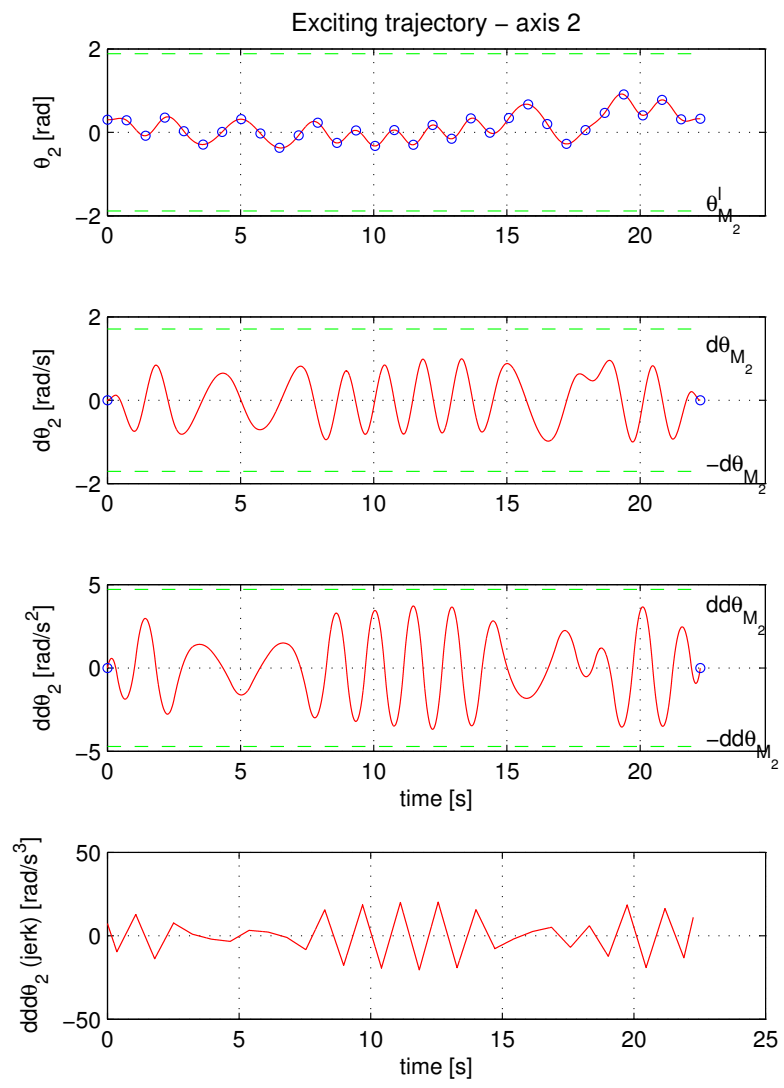

Fig. 3. Optimized trajectory for axis 2

The joint position and torque data were sampled with $1 \mathrm{~ms}$. To overcome the problem of noisy measurement data, some filter strategies were used as presented in [3] and [5]. For this purpose, the position data were filtered using a zerophase digital IIR lowpass butterworth filter in both direction with a cut-off frequency of $30 \mathrm{~Hz}$. The torque data with high frequency torque ripple was filtered similarly. The filtering procedure was processed with the Matlab decimate function.

\section{A. Estimated Parameters}

The estimated dynamic base parameters for the LWR are listed in Tab. I. As you can see from the values of relative standard deviation, most parameters are sufficiently estimated after the criterion given at the end of Sec. IID. Especially all parameter with a high influence on the dynamics are well estimated. You can see a slight trend on small valued parameters to decline in the quality of estimation. Due to the low influence to the dynamics this effect might be tolerated.

TABLE I

IDENTIFIED INERTIA BASE PARAMETERS OF LWR4 AND RELATIVE STANDARD DEVIATION. PARAMETER VALUES ARE EXPRESSED IN $\left[\mathrm{kgm}^{2}\right]$

\begin{tabular}{|c|c|c|}
\hline Parameter & Value & $\sigma_{\Phi_{r}} \%$ \\
\hline \hline ZZ1 & 0.0057 & 0.429 \\
\hline XX2 & 0.0057 & 1.523 \\
\hline YZ2 & 0.0583 & 0.824 \\
\hline ZZ2 & 0.0005 & 4.237 \\
\hline XX3 & 0.0573 & 2.458 \\
\hline YZ3 & 0.0331 & 1.019 \\
\hline ZZ3 & 0.0005 & 3.287 \\
\hline XX4 & 0.0054 & 4.367 \\
\hline YZ4 & 0.0516 & 0.994 \\
\hline ZZ4 & 0.0005 & 2.597 \\
\hline XX5 & 0.0004 & 6.579 \\
\hline YZ5 & 0.0506 & 1.354 \\
\hline ZZ5 & 0.0289 & 4.322 \\
\hline XX6 & 0.0004 & 7.324 \\
\hline ZZ6 & 0.0033 & 2.328 \\
\hline ZZ7 & 0.0001 & 1.397 \\
\hline
\end{tabular}

\section{B. Model Validation}

The quality of the obtained dynamic parameters is verified with a different validation trajectory. This trajectory was also built with B-splines, but the points were chosen randomly. After filtering the signal data as described in IV, torque differences were calculated both for torques predicted with the estimated dynamic parameters and torques predicted with the CAD data with respect to the measured torques. In Fig. (4) you can see the torque differences as error levels with their occurence over the measured validation trajectory for the joints 2-7. For joint 2 to 6 the torque errors show clearly decreased. Only for joint 7 the error occurence seems to be equal for both models, but in terms of the error level of about $1 \mathrm{Nm}$ this can be accepted.

\section{DISCUSSION AND CONCLUSION}

\section{A. Discussion}

The results in Tab. I show that the presented method using a B-spline parameterisation for the optimization problem can be used for the identification of dynamic parameters. From Fig. (4) it is evident that the estimated model has significantly more data points with lower error values than the CADvalued model. The use of torque sensors improves the torque data compared to the method of indirectly obtaining the 

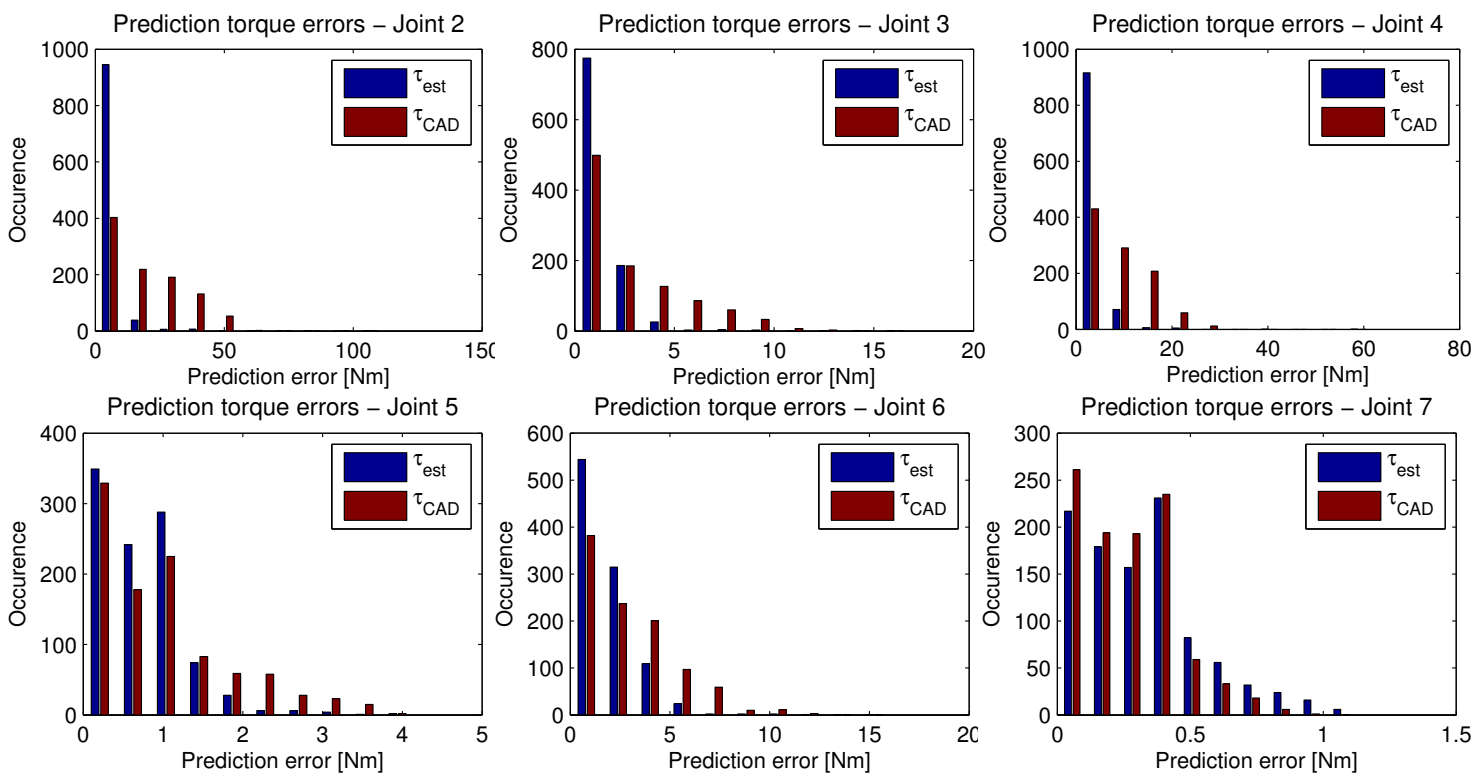

Fig. 4. Error occurence (torque difference) for a validation trajectory between the measured torques and the predicted torques of estimated model or CAD model respectively

torques via noisy measurements of motor current. Since the presented method is formulated as an optimization problem with strict constraints, the robustness of the formulation is much higher than that which uses the interpolation with $5^{\text {th }}$ order polynomial functions with a trial-and-error solution as presented e.g. in [2] and [3]. The method of using trajectories composed of a finit sum of harmonic sine and cosine functions presented in [6] is able to control the bandwidth of the periodic trajectories in order to avoid critical frequencies of the robots flexibilities. To avoid such critical frequencies with the method presented here, the obtained exciting trajectory could be analysed after the optimization with an FFT method.

\section{B. Conclusion}

In this paper we addressed the problem of finding optimum exciting trajectories for the identification of robot link inertia parameters. For this purpose a constraint nonlinear optimization problem was formulated and solved using a trajectory parameterization with B-splines. In the experimentation a DLR Light-Weight Robot with torque sensors in each of the 7 joints was used. A validation of the estimated model with a different validation trajectory shows clearly improvements on the accurray compared to a CAD-valued model.

\section{ACKNOWLEDGMENTS}

The authors would like to thank Andreas Stemmer and Thomas Hulin for their excellent work in carrying out the experiments on the robot.

\section{REFERENCES}

[1] F. Pfeiffer and J. Hölzl, "Parameter identification for industrial robots," in ICRA, 1995, pp. 1468-1476.

[2] M. Gautier and W. Khalil, "Exciting trajectories for the identification of base inertial parameters of robots," The Inernational Journal of Robotics Research, vol. 11, no. 4, pp. 362-375, 1992.
[3] W. Khalil, M. Gautier, and P. Lemoine, "Identification of the payload inertial parameters of industrial manipulators," in IEEE International Conference on Robotics and Automation - ICRA'07, 2007, pp. 49434948.

[4] C. Presse and M. Gautier, "New criteria of exciting trajectories for robot indentification." in ICRA (3)'93, 1993, pp. 907-912.

[5] M. Gautier, "Dynamic identification of robots with power model," in Proc. IEEE Int. Conf. on Robotics and Automation, April 1997, pp. 1992-1927.

[6] J. Swevers, C. Ganseman, D. Tükel, J. de Schutter, and H. V. Brussel, "Optimal robot excitation and identification," IEEE Transactions on Robotics and Automation, vol. 13, no. 5, pp. 730-740, 1997.

[7] C. Atkeson, C. H. An, and J. M. Hollerbach, "Rigid body load identification for manipulators," in Proc. of 24th Conference on Decision and Control, 1985, pp. 996-1002.

[8] P. Khosla and T. Kanade, "Parameter identification of robot dynamics," in Proc. of 24th IEEE Conference on Decision and Control, December 1985, pp. 1754-1760.

[9] G. Liu, K., K. Iagnemma, S. Dubowsky, and G. Morel, "A base force/torque sensor approach to robot manipulator inertial parameter estimation," in Proc. IEEE Int. Conf. on Robotics and Automation, May 1998, pp. 3316-3321.

[10] C. Atkeson, C. H. An, and J. M. Hollerbach, "Estimation of inertial parameters of manipulator loads and links," The Inernational Journal of Robotics Research, vol. 5, no. 3, pp. 101-119, 1986.

[11] J. C. Craig, Introduction to Robotics, 3rd ed. Perason Education, 2007.

[12] W. Khalil and E. Dombre, Modelling, Identification and Control of Robots. Hermes Penton Science, 2002.

[13] B. Armstrong, "On finding 'exciting' trajectories for identification experiments involving systems with non-linear dynamics," I. J. Robotic Res., vol. 8, no. 6, pp. 28-48, 1989.

[14] J. Hollerbach, W. Khalil, and M. Gautier, Springer Handbook of Robotics. Springer-Verlag Berlin Heidelberg, 2008.

[15] W. Khalil and F. Bennis, "Symbolic calculation of the base inertial parameters of closed-loop robots." I. J. Robotic Res., pp. 112-128, 1995.

[16] M. Gautier, "Numerical calculation of the base inertial parameters," Journal of Robotics Systems, vol. 8, no. 4, pp. 485-506, 1991.

[17] L. Biagiotti and C. Melchiorri, Trajectory Planning for Automatic Machines and Robots. Springer-Verlag Berlin Heidelberg, 2008. 